\title{
SEMILLERO DE INVESTIGACIÓN EN NANOTECNOLOGÍA (SINTEC) FACULTAD DE INGENIERÍA PROGRAMA DE INGENIERÍA ELECTRÓNICA UNIVERSIDAD AUTÓNOMA DE COLOMBIA
}

\author{
Fredy Alberto Cuéllar Torres \\ Ingeniero Electrónico, Fundación Universidad Autónoma de Colombia, \\ Líder e investigador del Semillero de Investigación en Nanotecnología (SINTEC). \\ fredy.cuellar@fuac.edu.co \\ Jorge Enrique López Duarte \\ Ingeniero Electrónico, Esp., Universidad Autónoma de Colombia, \\ Investigador del Semillero de Investigación en Nanotecnología (SINTEC). \\ jorge.lopez@fuac.edu.co \\ Javier Enrique Arévalo Peña \\ Ingeniero Electrónico, Esp., MSc. Fundación Universidad Autónoma de Colombia, \\ Investigador del Semillero de Investigación en Nanotecnología (SINTEC). \\ javier.arevalo@fuac.edu.co
}

Recibido: 06-11-2015, aprobado: 09-02-2016, última versión: 09-02-20161

\section{RESUMEN}

En el presente artículo se realiza una breve historia del Semillero de Investigación en Nanotecnología (SINTEC), de la Facultad de ingeniería del programa de ingeniería electrónica, donde se muestra como el grupo ha realizado y participado a nivel nacional e internacional representando a la Fundación Universidad Autónoma de Colombia en congresos, seminarios, encuentros de semilleros de Investigación, ganando premios y reconocimientos, cuyo objetivo es el de fortalecer la investigación en el campo Nanoescalar en la Universidad.

Palabras claves: Ciencia, Educación, Investigación, Nanotecnología, Semilleros, Universidad.

\section{ABSTRACT}

In this paper a brief history of Seed Research is done in Nanotechnology (SINTEC), Faculty of Engineering, which shows how the group has conducted and participated at national and international level representing the Universidad Autónoma de Colombia, at conferences, seminars, meetings of seed research, winning awards, whose goal is to strengthen research in the field at the University nanoscale.

Keywords: Science, Education, Research, Nanotechnology, Seeds, University. 


\section{INTRODUCCIÓN}

$\mathrm{L}$ a Nanociencia y Nanotecnología se ha convertido en una aliada permanente de la ciencia, de la Ingeniería, se evidencia la necesidad de conocer e interpretar y generar aplicaciones con el objetivo de ayudar a la calidad de vida de las personas.

La nanotecnología por su gran campo de acción despierta el interés de estudiantes, investigadores y científicos a desarrollar prototipos en áreas principales como lo es la ingeniería, a tal punto de la fabricación de Ordenadores Cuánticos, Nanotransistores, Pantallas Flexibles, Baterías de Larga Duración, memorias, entre otros, en áreas como infraestructuras se representan materiales muchos más fuertes y campos como el diseño en ropa aborda textiles inteligentes que no se arrugan, que no se mojan hasta el cambio de color [1].

La palabra nanotecnología es usada ampliamente para definir las ciencias y técnicas que se aplican a un nivel nanoescalar, que oscila entre 1 y 100 nanómetros. Por debajo de este nivel se encuentra la escala atómica, próxima a 0.1 nanómetros $(10-10 \mathrm{~m})$, seguida de la escala nuclear, cercana al femtómetro $(10-15 \mathrm{~m})$. Estas son medidas extremadamente pequeñas que permiten trabajar y manipular estructuras moleculares y atómicas [2].

Teniendo en cuenta la anterior de definición el Semillero de Investigación en Nanotecnología de la Facultad de Ingeniería se ha involucrado en el desarrollo de dispositivos semiconductores a escala nanométrica y prototipos teóricos basados en resultados de simulación que se han permitido obtener en este campo. Con esfuerzo, dedicación y por amor a la ciencia, la tecnología y con el gran sueño de que la Universidad siga cultivando estos caminos de investigación como lo es la Nanoescalar.

\section{LA CREACIÓN DEL SEMILLERO DE INVESTIGACIÓN EN LA UNIVERSIDAD Autónoma de Colombia}

En el mes de febrero del año 2009 ante el Sistema Unificado de Investigaciones (SUI), se genera el formato de inscripción de semilleros de investigación y desde ese momento se radica la creación del grupo ante La Universidad y el programa de Ingeniería Electrónica.

En este documento se especifica la misión, visión y los objetivos del semillero tal como se observa a continuación:

\section{A. Misión}

Ser un grupo consolidado que basados en nuestros conocimientos cumpla a cabalidad con los objetivos definidos por el mismo. Establecer alianzas estratégicas en el campo del desarrollo tecnológico ambiental para la solución de problemas que aquejan a nuestra sociedad. Participar con trabajos y resultados investigativos en eventos locales, nacionales e internacionales.

\section{B. Visión}

Nos proyectamos como un grupo que está a la vanguardia a los diferentes avances en el campo de la nanotecnología, para una futura aplicación de la electrónica en la innovación de herramientas para el trabajo de la nanotecnología.

\section{Objetivo general}

Generar y aplicar conocimientos al desarrollo de proyectos orientados a solucionar problemas de la industria nacional y la sociedad en general, relacionados con los campos de nanotecnología y nanoelectrónica.

\section{Objetivos específicos}

- Dar a conocer el grupo de investigación a nivel local, nacional e internacional, por 
sus diferentes aportes a la ciencia y a la comunidad educativa.

- Lograr publicaciones para diferentes revistas científicas mostrando los resultados obtenidos por el grupo.

- Alcanzar los máximos resultados con el grupo con el fin de lograr la obtención de un producto tangible y útil para las demás instituciones y comunidades educativas.

\section{E. logo}

El logo del Semillero de Investigación en Nanotecnología, Figura 1, representa un Buckybally en el centro una partícula encapsulada dentro de la estructura.

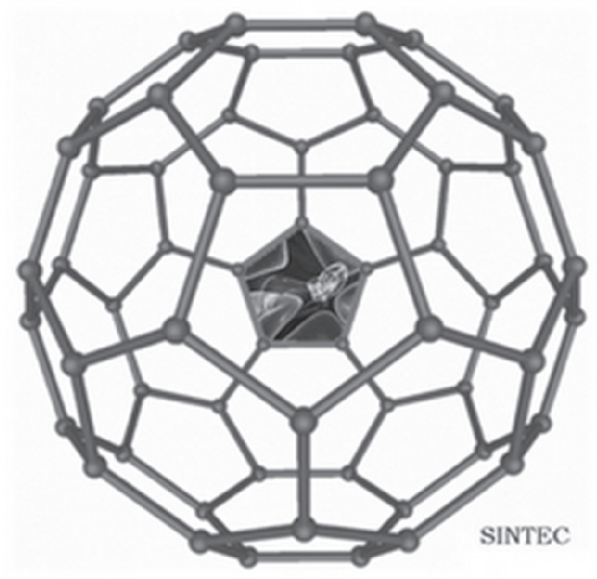

Figura 1

Logo del Semillero de Investigación

Cabe resaltar que los esfuerzos de los investigadores al inicio de las actividades del semillero fueron de gran compromiso, y buena voluntad, donde poco a poco se fue fortaleciendo a tal punto de ser un grupo de reconocimiento internacional y a nivel nacional ha tenido gran participación con Universidades de gran prestigio como lo son: La Universidad Nacional, La Universidad de los Andes y la Pontificia Universidad Javeriana.

\section{PARTicipación del SEMILLERo de INVESTIGACIÓN A EVENTOS Y REDES CIENTÍFICAS}

El semillero de investigación se ha distinguido en evento nacionales como Internacionales, se tiene en cuenta que en cada uno de ellos se ha participado como ponente, los cuales se destacan los siguientes.

\section{A. Eventos nacionales}

Gracias a todo el grupo de trabajo del Sistema Unificado de Investigaciones el semillero ha participado activamente en la Red Colombiana de Semilleros de Investigaciones (REDCOLSI), el cual tiene como objetivo realizar un encuentro de investigación de Universidades, este consta de un evento Regional que se desarrolla al principio del año, en este evento se reúnen todos los semilleros de investigación nivel Bogotá, en donde son evaluados, solo si se consigue el puntaje necesario se puede participar a nivel nacional, en donde nuevamente se realiza una evaluación para participar en el evento Internacional.

En orden cronológico el semillero ha asistido a los siguientes encuentros:

1. En el X Encuentro Regional de Semilleros de Investigación, el tipo del evento fue regional, de ámbito nacional que tuvo lugar en la Universidad Central Bogotá, los días 9, 10, 11, 12 de mayo del año 2012.

2. En el XV Encuentro Nacional y IX internacional de Semilleros de Investigación, el tipo del evento fue Nacional e Internacional, en la Universidad Cooperativa de Colombia Bucaramanga, los días 11, 12, 13, 14 de octubre del año 2012.

3. En el IX Encuentro Regional de Semilleros de Investigación, el tipo del evento fue Regional, en la Universidad San Buenaventura los días 13, 14, 15, de mayo del 2013. 
4. XI Encuentro Regional de Semilleros de Investigación, el tipo del evento fue regional, en la Universidad Manuela Beltrán los días 09, 10, 11 de Mayo del 2013, Universidad Manuela Beltrán, Bogotá, Colombia.

5. XII Encuentro Regional de Semilleros de Investigación, fue de tipo Regional y ámbito nacional, los días 9, 10, 11, de septiembre del 2014, Universidad Los Libertadores, Bogotá, Colombia

6. XIII Encuentro Regional De Semilleros De Investigación, el tipo del evento fue regional, los días 6, 8, 9 del año 2015, en la Universidad San Buenaventura.

7. Debido a que el programa de ingeniería Electrónica hace parte de la Red de Programas de Ingeniería Electrónica (REDIE), el semillero participo en calidad de ponencia al II Encuentro Nacional de Semilleros de Investigación en ingeniería, que tuvo lugar el 4 y 5 de noviembre de 2015 en la Universidad Central, con dos artículos de investigación denominados: Desarrollo de dispositivos semiconductores a escala nanométrica por medio de simulación empleando la herramienta ABACUS y Nanoscale Semiconductor Elements Design using the nanoHUB Software Tool.

\section{B. Eventos internacionales}

Los eventos más representativos que tuvo participación el Semillero de Investigación en Nanotecnología fueron los siguientes:

1. En el XII Encuentro Nacional y VI Internacional de Semilleros de Investigación (REDCOLSI) de Ámbito Internacional y Nacional, los días 8, 9 y 10 de noviembre del año 2008, en la Universidad Agustiniana, Bogotá.

2. En el Seminario internacional de nanociencia 2010, el cual tuvo lugar en la Sala de eventos del Hotel Dann Carlton, los días 21, 22, y 23 de octubre del año 2010, organizado por la Fundación Latinoamericana para la Promoción De La Ciencia (FUNLACI), ver Figura 2.

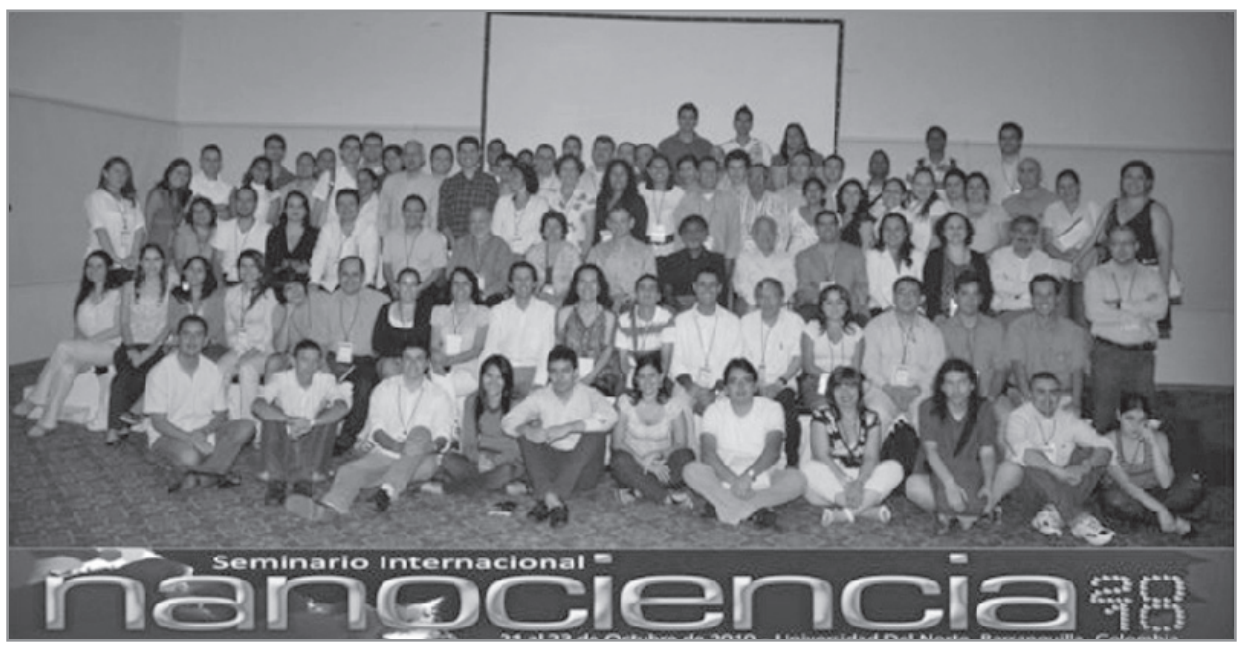

128

Figura 2

Seminario Nanociencia 2010

3. En el Congreso Internacional de Aplicaciones de la Nanotecnología, de ámbito Internacional que tuvo lugar el 8 de agosto del año 2011, en la sala de eventos de la Biblioteca Luis Ángel Arango, organizado por la Universidad de Cundinamarca. 
4. En el IV Seminario Internacional de Nanociencias y Nanotecnologías de ámbito internacional los días 17, 18, 19, 20, 21 de septiembre del año 2012, en el Palacio de Convenciones de la Habana-Cuba, organizado por el Comité científico del Estado de Cuba. Se resalta que la ponencia fue evaluada por científicos cubanos expertos en el área y organizados por el Científico Fidel Castro Hijo, ver Figura 3.

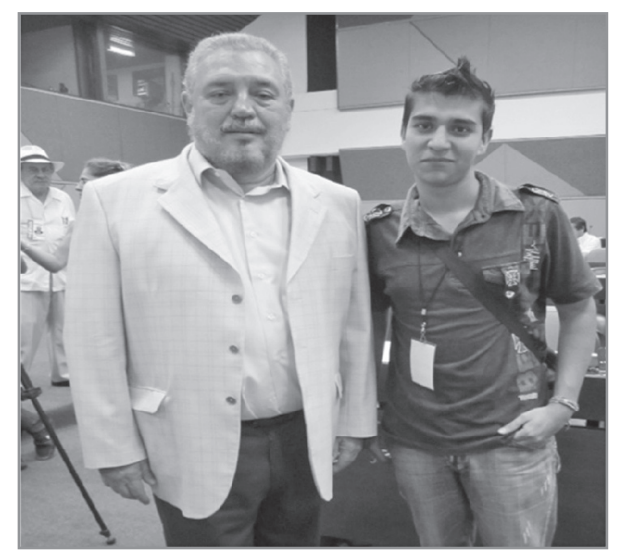

Figura 3

Izquierda Fidel Castro Hijo, Derecha Fredy Cuéllar (SINTEC)

En el anterior evento participaron Universidad Colombianas como: La Nacio- nal- Medellín, Universidad Distrital y con esfuerzo y dedicación la buena participación de la Universidad Autónoma de Colombia.

5. El semillero de Investigación participo en la Semana de Nanociencia y Nanotecnología donde tuvo lugar los siguientes eventos: III Simposio Iberoamericano de Divulgación y Formación en Nanotecnología - NANODYF'2014, III Congreso Nacional de Nanotecnociencias, III Congreso Nacional de Polímeros, y el XIV Taller de Desechos Agroindustriales y Marinos ADAM, 2014, en este evento participaron 4 Universidades Colombianas:

- Universidad Nacional de Colombia.

- Universidad de Los Andes.

- La Pontificia Universidad Javeriana.

- La Universidad Autónoma de Colombia.

En la Figura 4, se observa el grupo de científicos participantes en el evento, en este se tuvo la participación de países como: España, México, Estados Unidos, Chile, Colombia, Venezuela, entre otros.

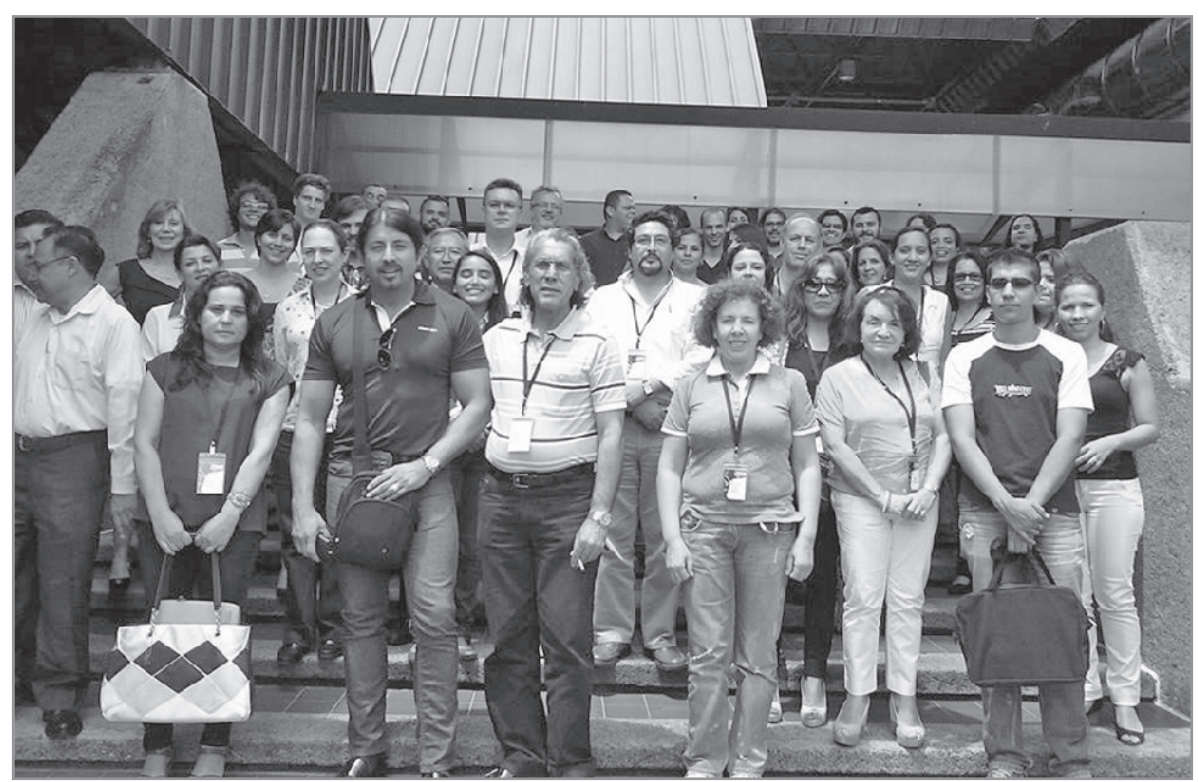

Figura 4

Participantes de la Semana de la Nanociencia y la Nanotecnología 


\section{Participación en la Red Nanocolombia}

El semillero de investigación en Nanotecnología en el mes de abril del año 2014 se unió a la Red Colombiana de Nanociencia y Nanotecnología.

El semillero activamente participo en el foro Nacional "Nanotecnología Medio Ambiente y Salud, Nanotecnología y el Problema de Contaminación por Mercurio" que tuvo lugar en la Pontificia Universidad Javeriana el 13 de junio del 2014, para este evento se participó con una Base de Datos, Materiales y Riesgo, Contaminación. Ver Figura 5, el cual está disponible en la página del Red Nanocolombia (http://www.rednanocolombia.org/).

Para seguir con la trayectoria con la Red Nanocolombia se participó en el II Foro "Nanotecnología para la sustitución y Mitigación del Uso del Mercurio en Procesos de Beneficio, Minería y
Medio Ambiente". Ver Figura 6, que tuvo lugar en la Universidad Pedagógica y Tecnológica de Colombia, Sede Sogamoso el 10 de octubre del año 2014.

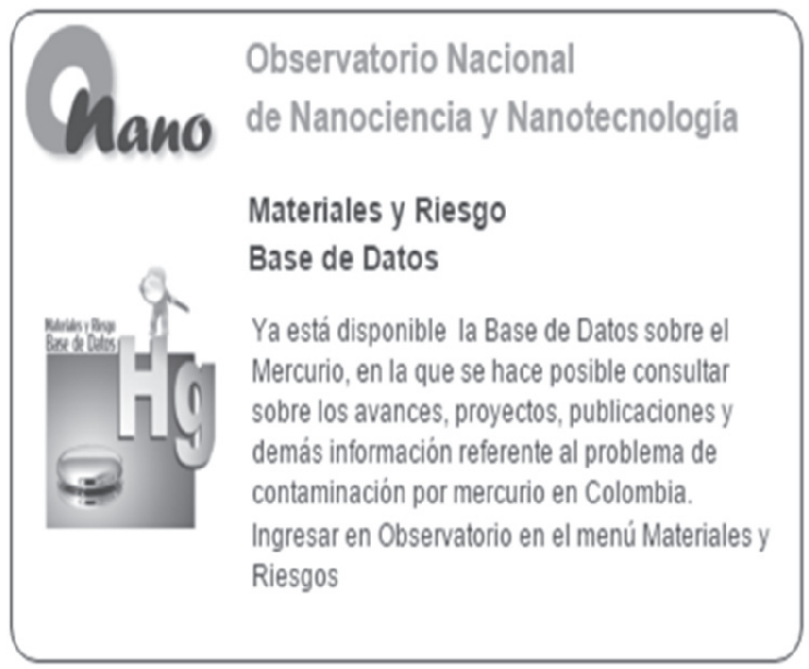

Figura 5

Base de Datos, tomada de (http://www.rednanocolombia.org/).

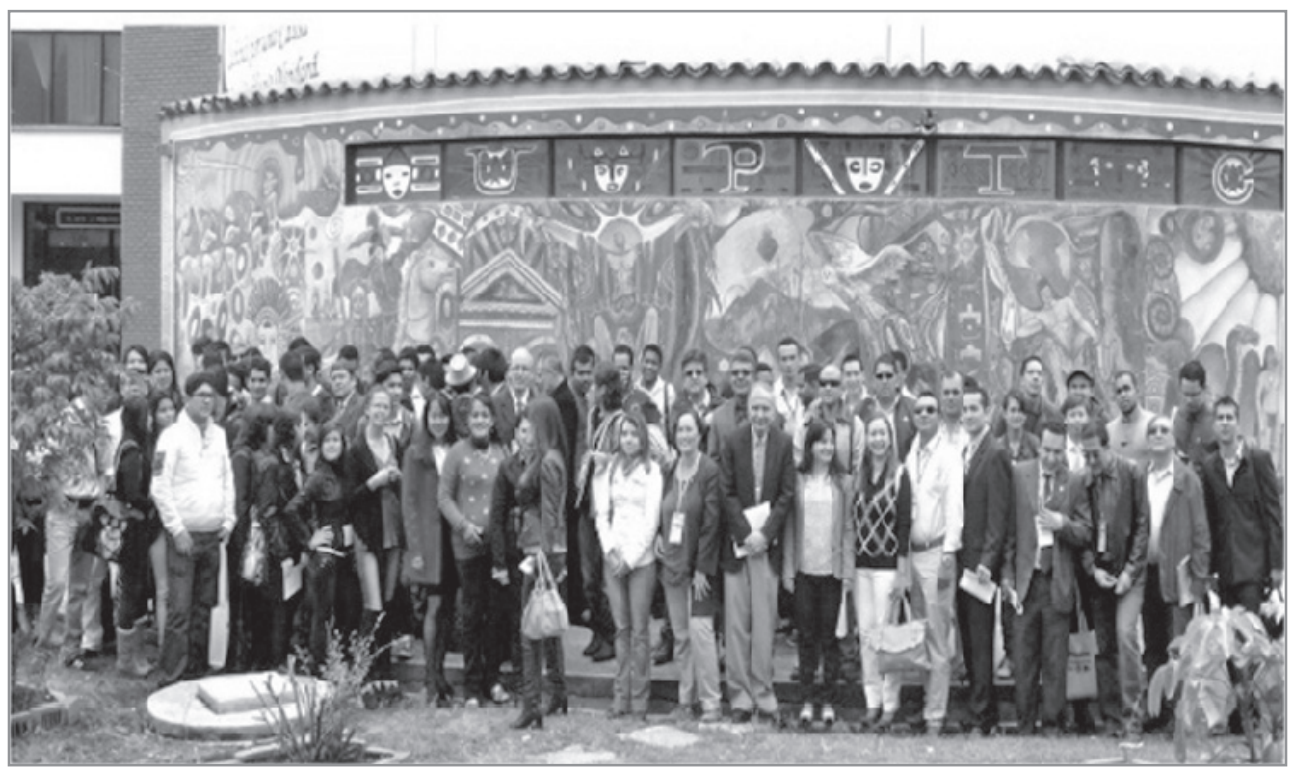

130

Figura 6

II Foro Nacional

Dentro de la buena participación del Semillero, fue invitado a la primera reunión de presentación del Consejo Asesor de la Red Nanocolom- bia, ver Figura 7, que tuvo lugar en la Academia de Ciencias 


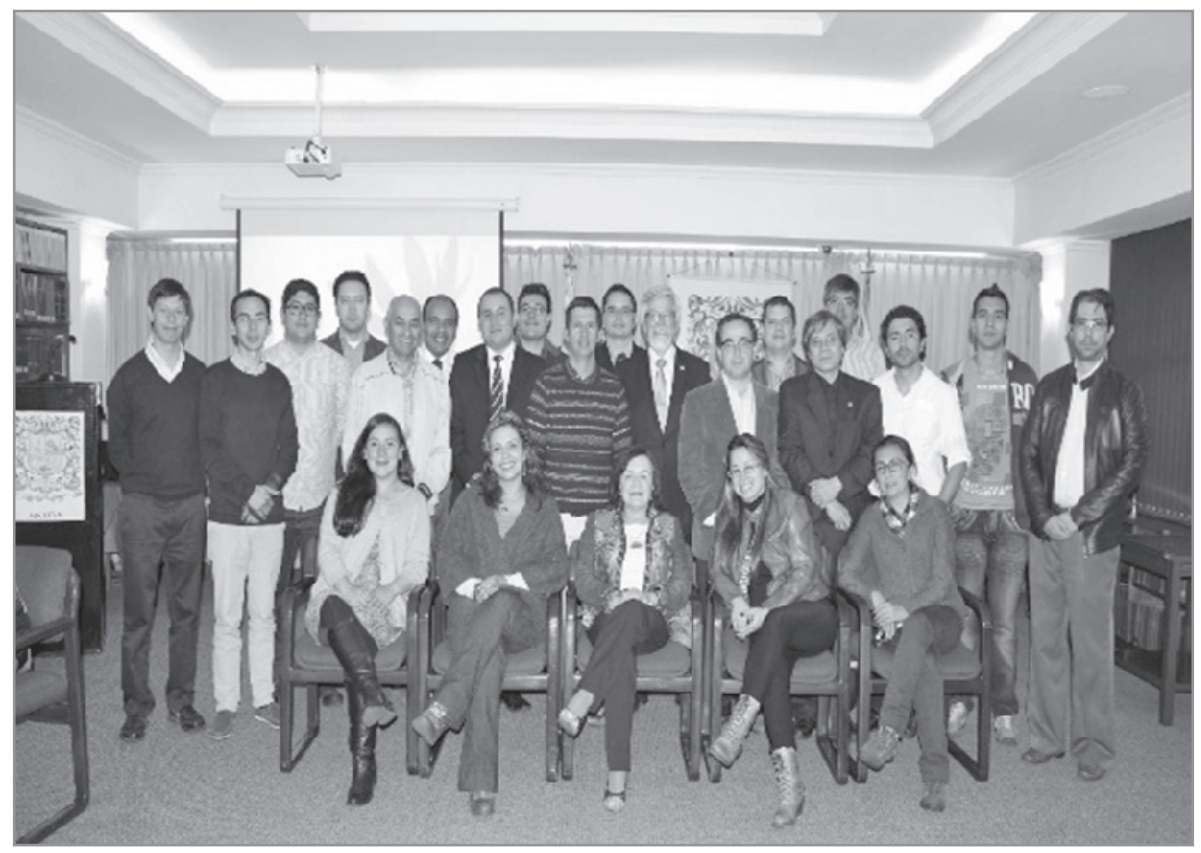

Figura 7

Primera Reunión consejo Asesor, Red Nanocolombia

Debido a las Buenas relaciones con La Red Colombiana de Nanociencia y Nanotecnología hemos tenido la oportunidad de Asistir a un curso de importancia internacional con el $\mathrm{PhD}$.
Rajender S Varma de la Agencia de la Protección Ambiental (EPA, por sus siglas en Ingles) de Estados Unidos, ver Figura 8.

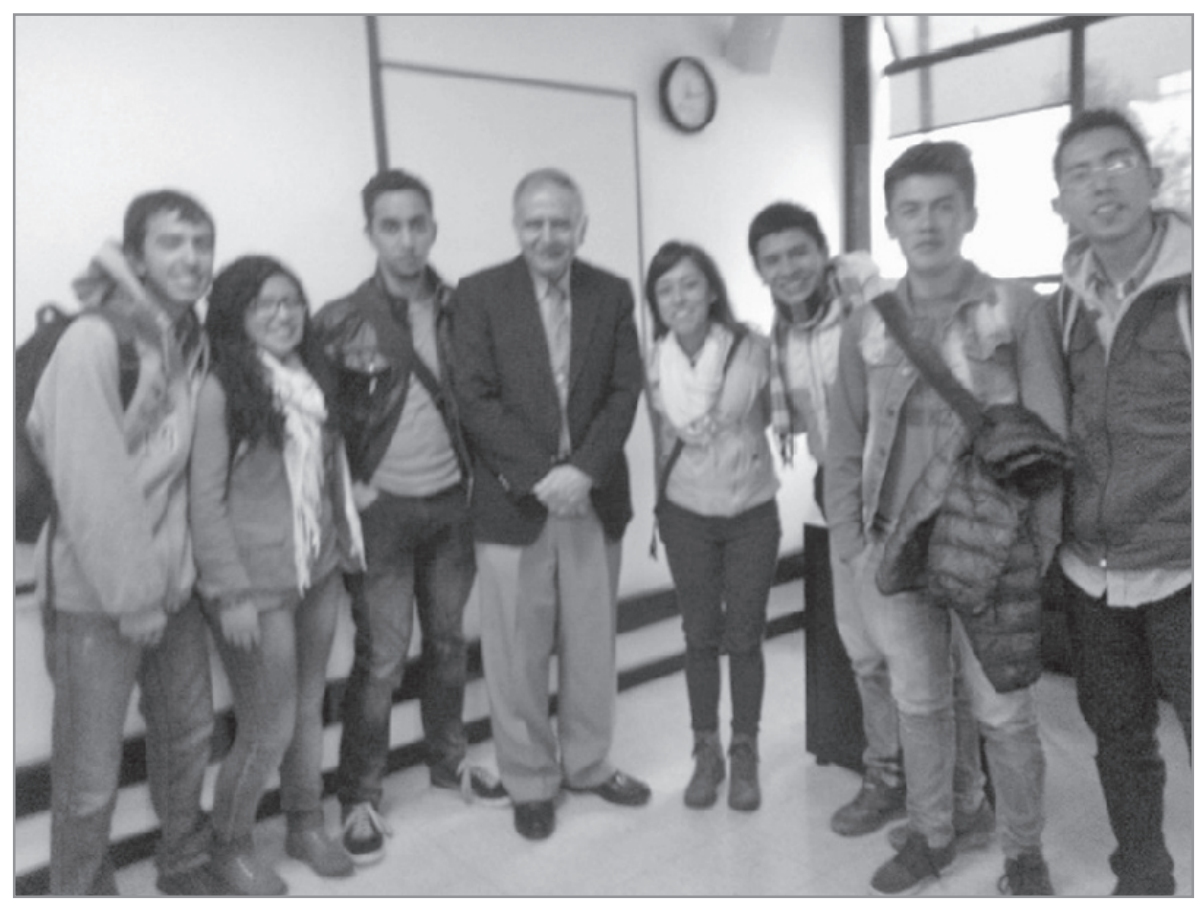

Figura 8

Semillero de Investigación SINTEC, junto con el científico Rajender S. Varma 


\section{RECONOCIMIENTOS OBTENIDOS}

- Uno de los reconocimientos más grandes del Semillero en Investigación en Nanotecnología (SINTEC) fue el ser nombrado en la revista Interdisciplinaria en Nanociencia y Nanotecnología, Mundo nano, Vol. 4, No. 2, julio-diciembre, 2011 de la Universidad Nacional Autónoma de México, donde el título del artículo es: La divulgación en Colombia de la Nanociencia y Nanotecnología en tiempos de Cambio, páginas 34-42, En este artículo se nombran las Universidades a nivel nacional que trabajan en el campo de la Nanotecnología

- Aval internacional para proyectos que fueron calificados como meritorios $y$ sobresalientes en el XVII Encuentro Nacional y XI Internacional de Semilleros de Investigación, realizado en la ciudad de Tunja, REDCOLSI, el cual el estudiante Fredy Alberto Cuéllar Torres obtuvo la calificación de proyecto meritorio con el proyecto "Aprendizaje de la Nanotecnología Para la Creación de Dispositivos Semiconductores, para ser presentado en el 1er. Congreso Latinoamericano y Centroamericano de Semilleros, Líderes y Grupos de Investigación, que tendrá lugar en abril del próximo año 2016.

- En el XIII Encuentro Regional De Semilleros De Investigación, el tipo del evento fue regional en el 2015, en la Universidad San Buenaventura, en donde se obtuvo la mención de proyecto sobresaliente para ser presentado en el XVIII Encuentro Nacional y XII Internacional de semilleros de investigación de REDCOLSI que se realizó del 8 al 11 de Octubre del 2015 en la Universidad Santiago de Cali.
El Semillero de Investigación en Nanotecnología SINTEC, agradece profundamente a la Uni- versidad Autónoma de Colombia por el apoyo brindado para las diferentes actividades realizados en el transcurso de cuatro años y medio, en especial agradece al Sistema Unificado de Investigaciones junto con el Director PhD. Julio Mario Rodríguez, las coordinadoras de investigación Ana Corena y Ximena Barbosa, en su momento el Decano Luis Héctor Peña y el Director de Carrera de electrónica Ing. Jorge Enrique López Duarte y al actual director de carrera Ing. Benjamín Rodríguez Vargas, al actual Decano Rafael Arturo Camerano Fuentes, al consejo de Facultad de Ingeniería y el consejo Directivo y en especial a la Doctora Delia Isabel Ayala Martínez, Directora de Pagaduría, por su apoyo al Semillero de Investigación.

\section{Conclusiones y ReCOMENDACIONES}

Para contextualizar el Semillero de Investigación cuenta participación en eventos, ver Tabla 1 , y unos de los resultados fue el primer trabajo de grado del semillero titulado “DESARROLLO DE DISPOSITIVOS SEMICONDUCTORES A ESCALA NANOMÉTRICA EMPLEANDO HERRAMIENTAS DE SIMULACIÓN", por el estudiante Fredy Cuéllar con una calificación 5.0.

Tabla 1

Participación en eventos

\begin{tabular}{|c|c|l|l|}
\hline No. & Cantidad & \multicolumn{1}{|c|}{ Descripción } & \multicolumn{1}{|c|}{ Impacto } \\
\hline 1 & 7 & $\begin{array}{l}\text { Eventos } \\
\text { Nacionales }\end{array}$ & Nacional \\
\hline 2 & 8 & $\begin{array}{l}\text { Eventos } \\
\text { Internacionales }\end{array}$ & Internacional \\
\hline 3 & 1 & Tesis de Grado & Nacional \\
\hline 4 & 1 & $\begin{array}{l}\text { Participación } \\
\text { en Redes }\end{array}$ & $\begin{array}{l}\text { Nacional e } \\
\text { Internacional }\end{array}$ \\
\hline
\end{tabular}

El semillero de investigación ha conseguido generar impacto y participación a nivel nacional en campo de la Nanociencia y Nanotecnología generando grandes contactos científicos, y La Universidad Autónoma de Colombia se 
ha convertido en un aliado en estos temas, los cuales los ha demostrado con reconocimientos y ponencias.

Se tiene en cuenta que la Universidad está enfocada en la Acreditación de alta calidad y conocemos la importancia de la Investigación, es por ello que uno de nuestros objetivos es realizar una exigente investigación, el cual nos ha permitido participar en el entorno científico a nivel nacional como internacional, comprometidos con el avance de la ciencia y aportar todo lo que sea necesario para el beneficio de la Universidad con nuestro camino en el campo de la Nanociencia y Nanotecnología.
Muchos de los resultados obtenidos en el Semillero de Investigación han sido obtenidos gracias a herramientas de investigación reconocidas como lo es Nanohub de la propiedad de La Universidad de Purdue, en donde se accedió a información teórica de científicos mundialmente conocidos como el PhD. Supriyo Datta.

Como recomendación final se hace necesario una posición fija en la Universidad que represente la investigación en el campo de la Nanociencia y La Nanotecnología, al igual un Laboratorio que permita obtener prototipos o dispositivos para poder ser aplicados.

\section{RefERENCIAS}

C. Martelo and D. Vinck (2009). "Redes Sociotécnicas de Cogestión de Conocimiento en Nanotecnologías en Colombia: ¿Entre la Visibilidad Internacional y la Apropiación Local?”, Redes, 15, 113.

Grünes A., R Saito, G. G. Samsonidze, M. A. Pimenta, A. Jorio, A. G. S. Filho, G. Dresselhaus, and M. S. Dresselhaus (2002). "Characterization of nanographite and carbon nanotubes by polarization dependent optical spectroscopy", MRS Proceedings, Symposium F.

M.C. Roco (2002). "National nanotechnology initiative", in In Outline of Research and Education Programs. Präsentation auf dem Workshop» The Nanomaterials and Chemical Industry R\&D Roadmap Workshop «der Chemical Industry Vision2020 Technology Partnership, 30, 10.

M. Lundstrom. “EE-612: Lecture 26: Heterostructure FETs”, Electrical and Computer Engineering, Purdue.

Red Colombiana de Nanociencia y Nanotecnología, Red Nanocolombia. Consultado (2015) el 5 de Nov. http://rednanocolombia.org/

"Workshop in Nanotechnolgy For Energy And Medical Applications", in First Colombia- US Workshop in Nanotechnolgy For Energy And Medical Applications, pp. ISBN 978-958-8790-45-9. 